\title{
地面三维激光扫描技术在变形监测中的应用
}

赵云飞

太原市勘察测绘研究院

DOI:10.18686/gmsm.v1i2.23

[摘 要] 本文首先简要介绍了地面三维激光扫描系统的发展背景及基本原理,详细阐述了地面三维激光扫描仪在变形监测 领域的数据集中处理流程和分析策略,并针对点云数据的具体特征,提出了点云模型及点云数据直接对比两种变形监测方 式。通过研发以激光点云数据为核心的变形分析软件, 设计两组对照试验, 验证两种变形分析方法的优劣性, 并测评激光扫描 技术在变形监测领域的实际应用价值。

[关键词] 地面三维激光扫描技术; 变形监测; 点云数据; 优劣性

\section{1 地面三维激光扫描技术发展背景}

我国地域辽阔, 物资储备丰裕, 但是部分区域由于地质结 构特殊, 极易发生山体滑坡、泥石流等自然灾害。为此, 加强对 灾害高频地段的监测成为专业科研人员致力研讨的新课题。

当下, 国内外对山体滑坡灾害采取的主要监测方法包括 环境突变监测、地质结构力学参数变化监测、地表变形监测 及深层结构位移监测等。其中, 应用范围较广泛的是地表变 形监测。经过大量理论研究与实践积累可知, 该监测方法的 应用日趋成熟, 并取得了良好的成效。

传统地表变形监测的基本原理是利用全站仪和全球定位 系统协调配合, 对重点区域进行持续不间断实时监测, 然而, 这 种监测手段所布设的监测点位有限, 所收集的数据信息量无法 满足实际需求, 使得实际监测工作落实不到位, 难以及时且准 确的预测山体滑坡灾害。另外, 由于部分监测区域自然环境复 杂, 增加了控制点布设的难度, 导致外业工作压力激增, 延长了 作业周期。随着地面三维激光扫描仪的推广应用, 利用高精密 度点云数据开展地表变形监测, 不仅提高了实际监测效率, 也 保证了灾害预测的精确性, 因而备受业内人士的推崇。

\section{2 简述三维激光扫描技术基本原理及分类}

\section{1 工作原理概括}

从专业角度来说, 三维激光扫描仪的工作原理与全站仪 的测距测角原理相似, 其主要是采用非接触方式, 通过发射 不同波长的激光束获取到物体表面的实际距离、水平或坚直 角度、反射强度等信息, 通过系统自动搜集、整合和存储, 生成点云数据。其中, 地面三维激光扫描仪针对物体所获取 的点云数据, 是以扫描测站坐标系为基准的。扫描测站坐标 系的基本概念是: 以激光束发射位置为坐标原点, 以扫描仪 主要的三个作业方向为 $X$ 轴、 $Y$ 轴和 $Z$ 轴。

在三维扫描坐标系内, 某一单点的坐标位置可表述为:

$$
\left\{\begin{array}{c}
\mathrm{X}=\mathrm{S} \cos \theta \cos \alpha \\
Y=S \cos \theta \sin \alpha \\
Z=S \sin \theta
\end{array}\right.
$$

其中, $S$ 代表监测点到坐标原点的实际距离, $\alpha$ 和 $\theta$ 分别 代表扫描仪水平坐标夹角和坚直坐标夹角。

\section{2 技术分类}

现阶段, 三维激光扫描仪被业内人士广泛应用, 按照系 统运行原理及与应用途径可将其划分为如下几类:

(1) 机载激光扫描系统

机载型激光扫描系统的结构组成主要包括激光扫描仪、 动态差分定位系统、摄像设备及计算机分析软件等。机载型 激光扫描系统可在有限时间内大量收集三维点云数据和影 像资料, 具有信息采集效率高、测量范围广等优势, 但基础配 套设备造价不菲, 且精确度低, 使得其在变形监测领域的应 用受到限制, 这也是业内专业人员一直致力攻克的难题。

(2) 车载激光扫描系统

车载激光扫描系统的核心是创建三维源数据模型, 主要 应用于城市安全维护和现代化建设领域。与机载激光扫描系 统相似, 其精确度有待商榷, 在变形监测领域的应用尚未普 及开来, 同时, 业内技术人员也正在寻求完善车载激光扫描 技术应用的策略。

(3) 便携式激光扫描系统

便携式激光扫描系统体量轻便、便于携带, 可满足对小 型对象扫描的需求。目前在室内扫描和精细物体建模领域应 用较多。

(4) 地面激光扫描系统

地面激光扫描系统的工作原理与全站仪有相似之处, 属 于固定式扫描系统。经过专业研究人员的大量理论探究与实 践积累, 地面激光扫描系统在变形监测领域应用的优势就是 精确度高、效率高。当下, 其不仅被应用在变形监测中, 在矿 区开采、隧道勘测领域也有所应用, 并取得了良好的成效。

3 在变形监测领域应用三维激光测量技术的具体流程

3.1 根据实际需求选择地面三维激光扫描仪

地面三维激光测量可以高效获取待测物体的点云数据, 不仅改善了作业效率, 节约了人力与时间成本, 也保证了数 
据的精确性。近年来, 地面三维激光扫描技术的应用逐步完 善, 并被拓展应用到多个领域。当下, 瑞格、天宝、莱卡等仪 器生产商为填补技术市场空缺, 推出了不同型号的设备, 其 参数、性能存在较大差异。为此, 根据实际需求选择恰当的 仪器设备至关重要。

山体滑坡变形监测需要测量的范围较广泛, 因此, 需要 采用测量较长的扫描仪设备。本次试验研究选用的是雷格公 司生产的 VZ-4000 扫描仪, 其最大扫描距离可达到 4000 米, 测量精度可控制在 10 毫米内, 扫描速率为每秒 30000 点, 可 满足一般变形监测的需求。

\section{2 统一点云数据坐标系}

地面三维激光仪扫描是在以观测基准站为中心的局部 坐标中, 获取待测物体的点云数据。在不同测站获取的点云 数据坐标参数并不统一。如果在扫描作业环节布设多个观测 基准站, 需要将所有站点获取的数据信息进行拼接, 统一到 同一坐标系中。现阶段, 针对统一坐标系应用频率较高的两 种策略为:

其一, 先确保多个基准观测站获取数据的精确性, 然后 布设有至少三个标靶的大地坐标, 将基准站数据统一转换到 大地坐标系中。

其二, 针对三个标靶分别设定大地坐标, 再将基准观测 站的数据直接转向各自的大地坐标系。基于变形监测作业范 围广泛, 经过深思熟虑, 本次试验研究采用第二种策略执行 坐标系的统一。

\section{3 针对点云数据进行去噪处理}

在测量作业过程中, 由于测量环境、基础配套设备、表 面涂层光线折射干扰等诸多因素的影响, 会产生噪声, 而这 些噪声会影响点云数据的集中处理。为确保监测作业的精确 性, 应当对源数据进行去噪处理。点云噪声滤波的基本工作 原理是以点云局部的法向量变化、 $\mathrm{K}$ 邻域数目以及点到局部 拟合曲面的距离等约束属性, 来判断某点是否属于孤立噪声 或随机噪声, 进而根据实际需求, 采用恰当的滤波处理工艺。, 由于孤立噪声具有邻域点少的特征, 在滤波处理环节, 可在 点云 $\mathrm{K}-\mathrm{D}$ 树索引基础上, 通过判断该点邻域范围内临近点数 量, 判断其是否为孤立点。

\section{4 分析形变量的方法}

\section{1 分析方法简介}

在应用三维激光扫描技术进行变形监测时, 需要在不同 时间段获取待测对象的点云数据, 进而通过对点云数据实行 综合对比, 观察相应的变化信息, 然后对这些数据信息展开 分析, 确定变形情况。

\section{2 对比点云数据}

直接对比点云数, 需要在不同时间段内获取待测对象的 点云数据, 然后对这些数据作差处理, 掌握被监测目标的具 体形变程度。应用频率较高的三种点云数据对比方法为: (1) 最佳拟合面; (2)豪斯多夫距离法; (3)平均距离法。

由于豪斯多夫距离法和平均距离法在实际应用过程中,
极易受数据质量的影响, 因此, 本实验采用最佳拟合面法进 行点云数据对比。此方法的具体步骤如下:

其一, 预先获取两个时期待测对象的点云数据, 之后选 择滤波处理技术实施去噪处理。其二, 构建具有目的性的牵 引结构, 提高数据索引效率。其三, 针对所选第二期的点, 在 上一期的 $K$ 邻域进行查找。其四, 借助邻域点绘制平面。

\section{3 构建点云数据模型}

相较于上文的点云数据对比法, 点云模型的特殊之处在 于多了一个中间环节, 就是在获取待测对象点云数据后, 要 依据这些点云数据构建相关立体模型, 通过对模型求差, 提 取被测目标的变量信息。在提取地形数据的过程中, 可以先 制作数字高程模型。具体步骤为:

(1) 获取待测对象必要的点云数据, 根据实际需求进行去 噪过滤处理, 排除干扰项, 提高数据的精确性。

(2)利用经过集中处理的点云数据构建模型。

(3)统一坐标系, 以前期数据建立的模型为评判模型, 使 用内插法将后期构建的数据模型导入评判模型中, 然后统一 坐标, 进而增强建模的精确性。

(4)根据模型所呈现的变化情况提取变量信息。

\section{5 对比试验分析}

选用两期模拟数据进行对比试验, 将一期数据作为墙面, 然后使用另外一期数据在墙面上模拟三处已存在形变的区 域。分别在墙面内放置三个物体一一本 2.5 厘米厚的书籍、 一个 4.5 厘米厚的盒子和一个 8 毫米厚的笔记本电脑, 以此 作为判断标准。

在分析这组点云数据变形程度的过程中, 选择点云数据 直接对比法, 其具体阈值设置为 $1.7 \mathrm{~cm}$ 。

从直观观测可知, 书籍与盒子的信息均可以快速提取出 来, 而较薄的笔记本电脑却无法被提取, 基于扫描仪设备的 精度值为 $1 \mathrm{~cm}$, 因此在设置阈值时, 需要使其大于扫面精度, 又因为笔记本厚度不足 1 厘米, 所以在该区域的信息无法通 过扫描仪呈现。

经过对比试验可以得出结论, 如果形变值未超出扫描仪 精度范围, 在实际测量中, 无法通过扫描仪呈现形变位置和 变化量。

\section{6 结束语}

综上所述, 将地面三维激光扫描技术应用在变形监测领 域, 不仅可以提高实际监测作业效率, 节约人力与时间成本, 还能最大限度的保证形变数据变化量, 为后续工作提供参考。

\section{[参考文献]}

[1]陈弘奕,胡晓斌,李崇瑞.地面三维激光扫描技术在变 形监测中的应用 [J].测绘通报,2014(12):74-77.

[2]刘奎源.变形监测中地面三维激光扫描技术的应用 [J].西部资源,2018(01):129-130.

[3]朱生涛.地面三维激光扫描技术在地形形变监测中的 应用研究[D].长安大学,2013(06):54. 